\title{
Identification of sequence polymorphisms in the mitochondrial deoxyribonucleic acid displacement-loop region as risk factors for systemic lupus erythematosus
}

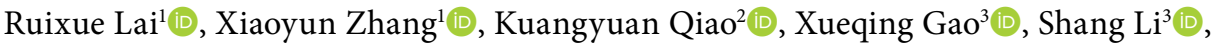

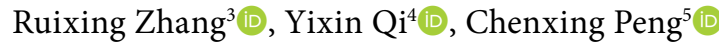 \\ ${ }^{1}$ Department of Immunology and Rheumatology, The Fourth Hospital of Hebei Medical University, Shijiazhuang, China \\ ${ }^{2}$ Basic Medical School, Hebei Medical University, Shijiazhuang, China \\ ${ }^{3}$ Department of Gastroenterology and Hepatology, The Fourth Hospital of Hebei Medical University, Shijiazhuang, China \\ ${ }^{4}$ Breast Center, The Fourth Hospital of Hebei Medical University, Shijiazhuang, China \\ ${ }^{5}$ Department of Immunology and Rheumatology, The Second Hospital of Hebei Medical University, Shijiazhuang, China
}

\begin{abstract}
Objectives: This study aims to evaluate the relationship between sequence polymorphisms (SNPs) in the displacement-loop (D-loop) region of mitochondrial deoxyribonucleic acid (mtDNA) and systemic lupus erythematosus (SLE) in Chinese female patients.

Patients and methods: This cross-sectional study was conducted between May 2017 and October 2017. The mtDNA was extracted from the peripheral blood of 97 female SLE patients (mean age 40.8 years; range, 20 to 79 years) and 108 age-matched healthy controls (mean age 48.7 years; range, 22 to 78 years). The SNPs of mtDNA D-loop were verified by polymerase chain reaction amplification and sequence analysis. The allele frequencies of D-loop region were compared by the Chi-square test between SLE and control groups.

Results: The SNP accumulation in SLE patients was significantly higher than that in the controls ( $p=0.027,95 \%$ confidence interval $[C l]: 0.075,1.210)$. The frequencies of the major alleles of the nucleotides $73 \mathrm{G} / \mathrm{A}(\mathrm{p}<0.001$, odds ratio $[O R]=1.241)$ and $195 \mathrm{~T} / \mathrm{C}(\mathrm{p}=0.047, \mathrm{OR}=4.318)$ as well as the minor allele of nucleotide 199T/C ( $p=0.048, O R=0.279)$ were significantly higher in the SLE patients than in the controls, which indicated that 73G, 195T and 199 C allele in the D-loop of mtDNA were associated with the risk of SLE. Further analysis indicated that the reactive oxygen species level in the SLE patients was significantly higher than that of controls (mean fluorescence intensity \pm standard deviation: $3054.333 \pm 256.099 \mathrm{vs} .2099 .167 \pm 599.662$, $\mathrm{p}=0.009,95 \% \mathrm{Cl}: 321.243,1589.091)$.
\end{abstract}

Conclusion: This study indicated the SNPs in the mtDNA may associated with the risk of SLE. Analysis of SNPs in the mitochondrial D-loop may help identify individuals who are at high risk of developing SLE.

Keywords: Displacement-loop, mitochondrial deoxyribonucleic acid, risk factors, sequence polymorphisms, systemic lupus erythematosus.

Systemic lupus erythematosus (SLE) is an autoimmune-mediated connective tissue disease characterized by the production of multiple autoantibodies and multi-system damage. The manifestations of SLE are notably diverse that range from rash, lupus encephalopathy, arthritis, glomerulonephritis, and hematological abnormalities. ${ }^{1,2}$ The incidence of SLE varies worldwide with 40-70 per 1,000 people in China; the incidence of SLE in females is nine times higher than that in males. ${ }^{3}$ Environmental, genetic, and hormonal factors may play roles in the disease, but the true mechanism underlying its development is still unclear. ${ }^{4}$ Increasing evidence has supported the importance of mitochondrial abnormalities which initiate the overproduction

Received: March 14, 2020 Accepted: June 11, 2020 Published online: December 14, 2020

Correspondence: Yixin Qi, MD and PhD. Breast Center, The Fourth Hospital of Hebei Medical University, Shijiazhuang, 050011, P.R. China. Tel: +8631186096288 e-mail: 13932153600@139.com 
of reactive oxygen species (ROS) and abnormal energy consumption in the pathogenesis of SLE. ${ }^{1,5}$

Mitochondria play essential roles in regulating ROS signaling, energy production, calcium homeostasis, and cell apoptosis. ${ }^{6}$ Human mitochondrial deoxyribonucleic acid (mtDNA) is a 16569-bp closed circular molecule encoding 13 proteins involved in the electron transport chain, two ribosomal ribonucleic acids (RNAs), and 22 transfer RNAs. The displacement-loop (D-loop) is a triple-stranded structure located in the main non-coding region that contains the main elements regulating the replication and expression of mtDNA..$^{6-8}$ Because of the high levels of ROS, a lack of protective histones, and limited DNA-restoration capacity, mtDNA is more prone to DNA mutations than nuclear DNA. ${ }^{7-10}$ Therefore, the SNPs of the mtDNA D-loop could affect the overall mitochondrial function so as to initiate genome damage, overproduction of ROS and nitrogen intermediates, abnormal energy expenditure, and auto-antigen production. ${ }^{1,5,11,12}$

The SNPs of mtDNA D-loop have been verified to have a predictive value for the risk of gastric carcinoma, colorectal cancer, and chronic kidney disease..$^{9,10,13}$ In this study, we aimed to evaluate the relationship between SNPs in the D-loop region of mtDNA and SLE in Chinese female patients.

\section{PATIENTS AND METHODS}

Between May 2017 and October 2017, blood specimens of 97 Chinese female patients (mean age: 40.8 years; range, 20 to 79 years) with SLE were obtained from the Department of Immunology and Rheumatology in the Second Hospital of Hebei Medical University for this cross-sectional study. Simultaneously, the blood specimens of 108 age-matched controls (mean age: 48.7 years; range, 22 to 78 years) who had no history of autoimmune diseases were collected. The diagnosis of SLE was based on the 1997 classification criteria for SLE by the American College of Rheumatology. ${ }^{14}$ The genomic DNA was extracted with the Wizard Genomic DNA extraction kit (Promega, Madison, WI, USA) based on the manufacturer's protocol and stored at $-20^{\circ} \mathrm{C} . .^{8,10,13}$ The study protocol was approved by the Second Hospital of Hebei Medical University Ethics Committee and supervised by the Human Tissue Research Committee (2017-P031). A written informed consent was obtained from each patient. The study was conducted in accordance with the principles of the Declaration of Helsinki.

The amplification of a 982-bp gene fragment located in the mtDNA D-loop region was performed using the forward primer 5'-CCCCATGCTTACAAGCAAGT-3' (nucleotides 16190-16209) and reverse primer 5'-GCTTTGAGGAGGTAAGCTAC-3' (nucleotides 602-583) with Polymerase Chain Reaction Master Mix Kit (Promega, Madison, WI, USA). ${ }^{9}, 10,13$ The target gene fragment was purified before sequencing. Cycle sequencing was performed using the dGTP BigDye ${ }^{\circledR}$ Terminator v3.0 Ready Reaction Cycle Sequencing Kit (Applied Biosystems, Foster City, CA, USA). The separation of products was performed using the ABI PRISM Genetic Analyzer 3730XL (Applied Biosystems, Foster City, CA, USA)..$^{8-10,13}$ The polymorphisms were verified by repeated analysis with both strands.

The ROS level was measured using BBOxiProbe ${ }^{\circledR}$ Plasma Active Oxygen Detection Kit (BestBio Technology, Shanghai, China) following the manufacturer's instructions. Briefly, $100 \mu \mathrm{L}$ plasma were incubated with $10 \mu \mathrm{L} \mathrm{O} 12$ probe for 30 minutes in the dark at $37^{\circ} \mathrm{C}$. The level of ROS was analyzed by Fluorescence Microplate Reader (BIOTEK, Winooski, VT, USA) at an excitation wavelength of $488 \mathrm{~nm}$ and an emission wavelength of $520 \mathrm{~nm}$. The experiments were repeated at least three times.

\section{Statistical analysis}

Paired and unpaired Student's t-tests were performed to determine the level of ROS, the distribution diversity of the D-loop SNPs, and the number of SNPs per patient among groups. The relationship of the clinical features and allele frequencies in D-loop region among the SLE patients was identified by the Chi-square test. Statistical analysis was performed using the IBM SPSS Statistics for Windows, version 21.0 software package (IBM Corp., Armonk, NY, USA). ${ }^{8-10,13} \mathrm{P}<0.05$ was considered to be statistically significant. 


\section{RESULTS}

The age distribution between the SLE patients and controls was not statistically significant (Table 1). A total of 130 SNP sites were detected within the mtDNA D-loop region of the controls and SLE patients. The fact that the average frequency of SNPs in each SLE patient was remarkably higher than that in the controls $(\mathrm{p}=0.027,95 \%$ confidence interval [CI]: $0.075,1.210)$ indicated that the SNPs were more accumulated in SLE patients (Table 2). The distribution frequencies for each allele of D-loop were compared by the Chi-square test between
SLE patients and controls. A total of 24 SNP sites with minor alleles frequency $>5 \%$ in both SLE patients and controls were analyzed. The frequencies of the major alleles of the nucleotides 73G/A ( $<<0.001$, odds ratio $[\mathrm{OR}]=1.241)$ and $195 \mathrm{~T} / \mathrm{C}(\mathrm{p}=0.047, \mathrm{OR}=4.318)$ as well as the minor allele of nucleotide 199T/C ( $\mathrm{p}=0.048$, $\mathrm{OR}=0.279$ ) were remarkably higher in the SLE patients compared with that of controls, which indicated that the patients carrying 73G, 195T, and $199 \mathrm{C}$ alleles were more susceptible to SLE (Table 3). As shown in Figure 1, further analysis indicated that the ROS level in SLE patients was significantly higher than that of controls

Table 1. Clinical characteristics of SLE patients and controls

\begin{tabular}{|c|c|c|c|c|}
\hline \multirow[t]{2}{*}{ Group } & $\begin{array}{l}\text { SLE patients } \\
\quad(n=97)\end{array}$ & $\begin{array}{l}\text { Controls } \\
(n=108)\end{array}$ & \multirow[b]{2}{*}{$\chi^{2}$} & \multirow[b]{2}{*}{$p$} \\
\hline & $\mathrm{n}$ & $\mathrm{n}$ & & \\
\hline $\begin{array}{l}\text { Age (year) } \\
\leq 50 \\
>50\end{array}$ & $\begin{array}{l}70 \\
27\end{array}$ & $\begin{array}{l}67 \\
41\end{array}$ & 2.365 & 0.124 \\
\hline \multicolumn{5}{|l|}{ Manifestations } \\
\hline Rash & 55 & - & & \\
\hline Ulcer & 8 & - & & \\
\hline Serositis & 8 & - & & \\
\hline Arthritis & 34 & - & & \\
\hline Hematological abnormalities & 43 & - & & \\
\hline Lupus nephropathy & 32 & - & & \\
\hline Anti-dsDNA (+) & 38 & - & & \\
\hline Lupus encephalopathy & 18 & - & & \\
\hline \multicolumn{5}{|l|}{ SLEDAI } \\
\hline$\geq 15$ (severe) & 16 & - & & \\
\hline 10-14 (moderate) & 24 & - & & \\
\hline 5-9 (mild) & 32 & - & & \\
\hline 0-4 (mildly inactive) & 25 & - & & \\
\hline \multicolumn{5}{|l|}{ Disease duration (years) } \\
\hline$\leq 1$ & 60 & - & & \\
\hline $1-5$ & 15 & - & & \\
\hline$>6$ & 22 & - & & \\
\hline \multicolumn{5}{|l|}{ Immunomodulatory medication use } \\
\hline Untreated & 49 & - & & \\
\hline Glucocorticoids & 15 & - & & \\
\hline Cyclophosphamide/+glucocorticoids & 6 & - & & \\
\hline Antimalarials/+glucocorticoids & 17 & - & & \\
\hline DMARDs/+glucocorticoids & 8 & - & & \\
\hline Biologics & 2 & - & & \\
\hline
\end{tabular}

SLE: Systemic lupus erythematosus; $\chi^{2}$ Chi-square; Anti-dsDNA: Anti-double stranded deoxyribonucleic acid; SLEDAI: Systemic lupus erythematosus disease activity index; DMARD: Disease-modifying antirheumatic drug, including azathioprine, methotrexate, and mycophenolate mofetil. 
Table 2. Average single nucleotide polymorphism frequency in the mitochondrial deoxyribonucleic acid displacement-loop for each group

\begin{tabular}{|c|c|c|c|c|c|}
\hline & SLE patients $(\mathrm{n}=97)$ & Controls $(n=108)$ & & & \\
\hline & Mean \pm SD & Mean \pm SD & $\mathrm{t}$ & $p$ & $95 \% \mathrm{CI}$ \\
\hline SNPs/patients & $8.402 \pm 1.977$ & $7.759 \pm 2.126$ & 2.234 & 0.027 & $(0.075,1.210)$ \\
\hline
\end{tabular}

SLE: Systemic lupus erythematosus; SD: Standard deviation; SNPs: Single nucleotide polymorphisms; CI: Confidence interval.

Table 3. Single nucleotide polymorphism sites showing frequency difference between SLE patients and controls

\begin{tabular}{|c|c|c|c|c|c|c|c|}
\hline \multirow{3}{*}{ Nucleotide } & \multicolumn{2}{|c|}{ SLE patients $(\mathrm{n}=97)$} & \multicolumn{2}{|c|}{ Controls ( $\mathrm{n}=108$ ) } & \multirow[b]{3}{*}{$\chi^{2}$} & \multirow[b]{3}{*}{$p$} & \multirow[b]{3}{*}{ OR } \\
\hline & Major & Minor & Major & Minor & & & \\
\hline & Allele & Allele & Allele & Allele & & & \\
\hline $73 \mathrm{G} / \mathrm{A}$ & $95^{*}$ & 0 & 87 & 21 & 20.604 & $<0.001$ & 1.241 \\
\hline 195T/C & 95 & 2 & 99 & 9 & 3.958 & 0.047 & 4.318 \\
\hline 199T/C & 88 & 9 & 105 & 3 & 3.918 & 0.048 & 0.279 \\
\hline
\end{tabular}

SLE: Systemic lupus erythematosus; $\chi^{2:}$ Chi-square; OR: Odds ratio; * Ninety-five systemic lupus erythematosus patients carry homozygous $\mathrm{G} / \mathrm{G}$ at nucleotide 73 , while two patients carry $\mathrm{C} / \mathrm{C}$ at this nucleotide position, but no $\mathrm{C}$ allele could be found in controls.

(mean fluorescence intensity \pm standard deviation: $3054.333 \pm 256.099$ vs. 2099.167 \pm 599.662 , $\mathrm{p}=0.009, \quad 95 \%$ CI: 321.243, 1589.091). No association was obtained between these SLE riskassociated SNPs and clinical characteristics based on our analysis (data not shown). The linkage disequilibrium analysis was also performed to analyze the interaction among SLE risk-associated SNPs, which revealed no association (data not shown).



Figure 1. Reactive oxygen species level of groups. SLE: Systemic lupus erythematosus.

\section{DISCUSSION}

In previous studies, the SNPs of mtDNA $\mathrm{D}$-loop have been verified to have a predictive value for the risk of a variety of diseases including hepatocellular carcinoma, gastric carcinoma, colon carcinoma, and chronic kidney disease. .10,13 $^{-13}$ However, few studies have evaluated the value of mitochondrial DNA variants for predicting SLE. In this case-control study, we found that the SNPs were more accumulated in SLE patients, and the patients carrying 73G, 195T, and 199C alleles were more susceptible to SLE.

However, the exact mechanism for the contribution of these SNPs to the risk of SLE is yet to be elucidated. The D-loop contains the main elements regulating the replication and expression of mtDNA. ${ }^{6-8}$ The accumulation of SNPs in the mtDNA D-loop region may modify the replication and expression of mtDNA so as to initiate abnormal energy expenditure and ROS overproduction. ${ }^{6,7}$ The oxidative DNA marker of 8-hydroxy-2'-deoxyguanosine was correlated with lupus nephritis.,15 In addition, ROS could initiate not only the formation 
of pro-inflammatory cytokines by activating nuclear factor kappa B signaling pathways but also the formation of SLE auto-antigens including the neutrophil extracellular trap and the apoptotic bleb.,11,12 Our data also show that patients with SLE suffer from a higher ROS level compared with that of matched controls. All of these implied that the SLE-associated SNP might modify the onset of SLE by initiating ROS overproduction.

All of these SLE-associated SNPs are located in the hypervariable segment 2 region, where somatic and germline mtDNA mutations occur preferentially. ${ }^{1}$ The $73 G$ allele has been identified as a risk-associated SNP for colon cancer, gastric cancer, and chronic kidney disease. ${ }^{9,10,13}$ In addition, the nucleotide 73G/A has been reported to be related to Leber's hereditary optic neuropathy and auditory neuropathy. ${ }^{16,17}$ The nucleotide 195T/C has been found to be correlated with Alzheimer's disease and colon cancer., ${ }^{918}$ The nucleotide199T/C has been found to be associated with coronary heart disease..$^{19}$ Consistent with the previous studies, the major alleles of nucleotides 73G/A and 195T/C as well as the minor allele of nucleotide 199T/C were confirmed as the risk-related SNPs for SLE in the present study.

This study has some limitations. The patients in this study were from a single-center institution and the findings presented should be verified by analyzing participants from different regions and of different ethnic groups. Furthermore, the exact mechanism for the contribution of these SNPs to the risk of SLE is yet to be elucidated.

In conclusion, this study indicated the SNPs in the mtDNA may associated with the risk of SLE. Analysis of SNPs in the mitochondrial D-loop may help identify individuals who are at high risk of developing SLE.

\section{Declaration of conflicting interests}

The authors declared no conflicts of interest with respect to the authorship and/or publication of this article.

\section{Funding}

This work was supported by the Natural Science Foundation of Hebei Province, China. (Grant No. H2019206428).

\section{REFERENCES}

1. Perl A. Oxidative stress in the pathology and treatment of systemic lupus erythematosus. Nat Rev Rheumatol 2013;9:674-86.

2. Tang Y, Wang L, Zhu M, Yang M, Zhong K, Du Q, et al. Association of mtDNA M/N haplogroups with systemic lupus erythematosus: a case-control study of Han Chinese women. Sci Rep 2015;5:10817.

3. Li M, Zhang $\mathrm{W}$, Leng $\mathrm{X}$, Li Z, Ye Z, Li C, et al. Chinese SLE Treatment and Research group (CSTAR) registry: I. Major clinical characteristics of Chinese patients with systemic lupus erythematosus. Lupus 2013;22:1192-9.

4. Carter EE, Barr SG, Clarke AE. The global burden of SLE: prevalence, health disparities and socioeconomic impact. Nat Rev Rheumatol 2016;12:605-20.

5. Lightfoot YL, Blanco LP, Kaplan MJ. Metabolic abnormalities and oxidative stress in lupus. Curr Opin Rheumatol 2017;29:442-9.

6. Andrews RM, Kubacka I, Chinnery PF, Lightowlers RN, Turnbull DM, Howell N. Reanalysis and revision of the Cambridge reference sequence for human mitochondrial DNA. Nat Genet 1999;23:147.

7. Herst PM, Rowe MR, Carson GM, Berridge MV. Functional Mitochondria in Health and Disease. Front Endocrinol (Lausanne) 2017;8:296.

8. Zhang R, Zhang F, Wang C, Wang S, Shiao YH, Guo $Z$. Identification of sequence polymorphism in the D-Loop region of mitochondrial DNA as a risk factor for hepatocellular carcinoma with distinct etiology. J Exp Clin Cancer Res 2010;29:130.

9. Guo Z, Zhao S, Fan H, Du Y, Zhao Y, Wang G. Identification of sequence polymorphisms in the D-Loop region of mitochondrial DNA as a risk factor for colon cancer. Mitochondrial DNA A DNA Mapp Seq Anal 2016;27:4244-5.

10. Wang H, Wang Y, Zhao Q, Guo Z, Zhang F, Zhao Y, et al. Identification of sequence polymorphisms in the D-Loop region of mitochondrial DNA as a risk factor for gastric cancer. Mitochondrial DNA A DNA Mapp Seq Anal 2016;27:1045-7.

11. Boilard E, Fortin PR. Connective tissue diseases: Mitochondria drive NETosis and inflammation in SLE. Nat Rev Rheumatol 2016;12:195-6.

12. Lee HT, Wu TH, Lin CS, Lee CS, Wei YH, Tsai CY, et al. The pathogenesis of systemic lupus erythematosus - From the viewpoint of oxidative stress and mitochondrial dysfunction. Mitochondrion 2016;30:1-7.

13. Bai Y, Guo Z, Xu J, Zhang J, Cui L, Zhang H, et al. Association of sequence polymorphism in the mitochondrial D-loop with chronic kidney disease. Ren Fail 2014;36:781-4.

14. Hochberg MC. Updating the American College of Rheumatology revised criteria for the classification of systemic lupus erythematosus. Arthritis Rheum 1997;40:1725. 
15. Shah D, Kiran R, Wanchu A, Bhatnagar A. Oxidative stress in systemic lupus erythematosus: relationship to Th1 cytokine and disease activity. Immunol Lett 2010;129:7-12.

16. Wang Q, Li R, Zhao H, Peters JL, Liu Q, Yang L, et al. Clinical and molecular characterization of a Chinese patient with auditory neuropathy associated with mitochondrial 12S rRNA T1095C mutation. Am J Med Genet A 2005;133A:27-30.

17. Zhao F, Guan M, Zhou X, Yuan M, Liang M, Liu Q, et al. Leber's hereditary optic neuropathy is associated with mitochondrial ND6 T14502C mutation. Biochem Biophys Res Commun 2009;389:466-72.

18. Coskun PE, Beal MF, Wallace DC. Alzheimer's brains harbor somatic mtDNA control-region mutations that suppress mitochondrial transcription and replication. Proc Natl Acad Sci U S A 2004;101:10726-31.

19. Qin Y, Xue L, Jiang $P, X u M, H e ~ Y$, Shi $S$, et al. Mitochondrial tRNA variants in Chinese subjects with coronary heart disease. J Am Heart Assoc 2014;3:e000437. 